Check for updates

Cite this: RSC Adv., 2017, 7, 24914

Received 29th March 2017 Accepted 23rd April 2017

DOI: $10.1039 / c 7 r a 03643 d$

rsc.li/rsc-advances

\section{Combined use of breath figures process and microphase separation of PS-b-P4VP to produce stable porous nanomaterials}

\author{
Baozhen Wu, ab Meimei Zhou, ${ }^{c}$ Wanlin Zhang, ${ }^{b}$ Yun Liang, ${ }^{b}$ Fengting Li ${ }^{\star a}$ \\ and Guangtao Li iD *b
}

\begin{abstract}
Among various templating strategies available for the preparation of porous polymer films, Breath Figures (BFs) as a fast, low-cost and versatile method has aroused extensive interest. However, the prerequisite of these porous polymer films for potential application is their stability in harsh environments. In this article, we report a new method to stabilise the porous materials prepared by BFs. Polystyrene-block-poly(4vinylpyridine) block copolymer (PS-b-P4VP) is utilized to prepare well-organized honeycomb films with pore diameters ranging from 0.45 to $2 \mu \mathrm{m}$ via BFs. Then ethanol is selected to swell the honeycomb porous membrane to generate a nanometer-sized microstructure by solvent induced microphase separation. After the microphase separation process by swelling in ethanol, pyridine moieties as hydrogen bond acceptor or alkaline catalyst are completely exposed, vitally important for the further improvement of film stability. On the one hand, monomers with hydrogen bond donors such as pyrrole, aniline, 3-thiopheneacetic acid can composite with the exposed pyridine groups by hydrogen bond interaction, and the subsequent chemical oxidation polymerization affords hierarchically porous conducting nanomaterials with thermal and solvent stability. On the other hand, the exposed pyridine groups can also be used as alkaline catalytic sites to hydrolyze inorganic precursor like TEOS on the skeleton of PS- $b$-P4VP for the final formation of silica inorganic porous films with prominently enhanced stability. All the results indicate that the combination of BF process and microphase separation is a versatile and effective method to produce various stable porous nanomaterials.
\end{abstract}

\section{Introduction}

As one of the most promising strategies for the fabrication of large-size films with ordered honeycomb arrays, breath figure (BF) is a fast, low-cost and facile method, first reported by François et al. in 1994. ${ }^{1}$ It is a bottom-up method, and the formation of breath figure films is a very complicated process. Up to now, no general mechanisms have been put forward to explain the experimental results. ${ }^{2}$ A well-accepted mechanism of breath figures is proposed as follows: (a) cooling of the solution and nucleation of the moisture, producing small but disordered water droplets on the solution surface; (b) growth and self-assembly of the water droplets, forming an ordered and closely packed water droplets array that covers the entire surface

${ }^{a}$ College of Environmental Science and Engineering, State Key Laboratory of Pollution Control and Resource Reuse, Tongji University, 200092 Shanghai, China. E-mail: fengting@tongji.edu.cn; Tel: +862165983302

${ }^{b}$ Department of Chemistry, Key Lab of Organic Optoelectronic and Molecular Engineering, Tsinghua University, 100084 Beijing, China. E-mail: lgt@mail. tsinghua.edu.cn; Tel: +861062792905

'Key Laboratory of Subsurface Hydrology and Ecological Effects in Arid Region, Ministry of Education, School of Environment Science and Engineering, Chang'an University, 710054 Xi'an, China of the solution; (c) evaporation of the solvent and water droplets, leaving a hexagonal pore array on the dry film. ${ }^{2,3}$ The regular porous films fabricated by breath figures have achieved more and more attention for their potential applications in many areas including templates, ${ }^{4}$ separation, ${ }^{5}$ sensors ${ }^{6}$ and biology $\mathrm{y}^{7}$ due to its low cost, time efficiency and ease of implementation.

Unfortunately, most films fabricated by breath figures so far are soluble in organic solvents and unstable under high temperatures. In order to be used for practical applications, fabricating thermally stable and organic solvent resistant porous films is an urgent demand. There are a lot of significant contributions to improve the film stability by the cross-linking approaches and other approaches. ${ }^{8}$ Bunz and co-workers reported a thermal-induced cross-linking strategy to stable the produced honeycomb films and the resultant films show high thermal stability. ${ }^{9}$ Besides many efforts have also been made on converting the breath figure films into inorganic materials to stable them. $\mathrm{Li}$ and co-workers reported the utilization of catalytically active inorganic honeycomb structures converted from hybrid films to fabricate inorganic nanorod arrays. ${ }^{\mathbf{1 0}}$ However, strategies to stabilize the porous films fabricated by BFs are still limited to specific materials. 
In this article, we report a new method to stabilize the BF films by microphase separation, and subsequent two approaches for film stability via chemical oxidative polymerization of organic molecules (Scheme 1) or the in situ growth of inorganic nanoparticles. Polystyrene-block-poly(4-vinylpyridine) (PS- $b$-P4VP) is a widely used amphiphilic block copolymer (BCP) and can make water droplets very stable during BF. Additionally, the diameter of the honeycomb hole can be adjusted by many factors, ${ }^{3}$ such as substrate and relative humidity. Here we studied the influence of volume fraction of PS- $b$-P4VP on structures of hierarchically porous films. The microphase separation happened after solvent evaporation and was shown when the entire film was put into non-solvent which cannot dissolve the polymer but can swell one block of the BCP, leading to the worm-like channel. After the microphase separation process by swelling in ethanol, all the pyridine moieties in the film are completely exposed which are hydrogen bond acceptors as well as alkaline catalyst. This is the key step for further stabilizing the porous films. On the one hand, monomers (pyrrole, aniline, 3-thiopheneacetic acid) as hydrogen bond donors are used to fabricate the hydrogen bond complexes and the subsequent chemical oxidative polymerization affords stable nanomaterials. After the removal of block copolymer by soaking the film in dichloromethane, the hierarchically porous structure generated by breath figures and microphase separation is preserved. Simultaneously the derived nanostructured conducting polymer films are obtained. Notably, the introduction of conductive polymers imparts the hierarchically polymer films with good chemical and thermal stability. On the other hand, the exposed pyridine moieties can also be used as alkaline catalyst for the hydrolysis inorganic precursors such as TEOS to realize the in situ growth of silica nanoparticles on the BF film after the microphase separation. Likewise, removing the block copolymer offers prominently stable inorganic porous film. Therefore, the proposed method here by the combined use of breath figures and microphase separation of PS- $b$-P4VP is very appealing to produce stable porous both organic and inorganic nanomaterials.

\section{Experimental section}

\section{Materials}

Styrene, 4-vinyl pyridine, 2,2-azobisisobutyronitrile (AIBN), ethanol, pyrrole, aniline, 3-thiopheneacetic acid, $\mathrm{FeCl}_{3}$, TEOS and chloroform were purchased from the chemical suppliers. All the chemical reagents were used without further purification unless otherwise stated. Styrene and 4-vinyl pyridine was distilled under reduced pressure and stored in refrigerator. AIBN was purified by
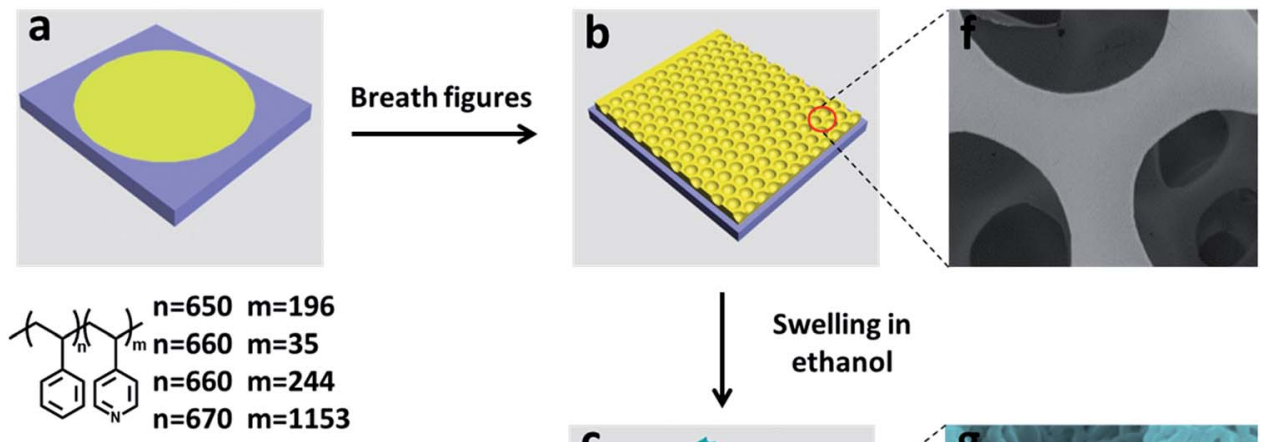

Swelling in

ethanol
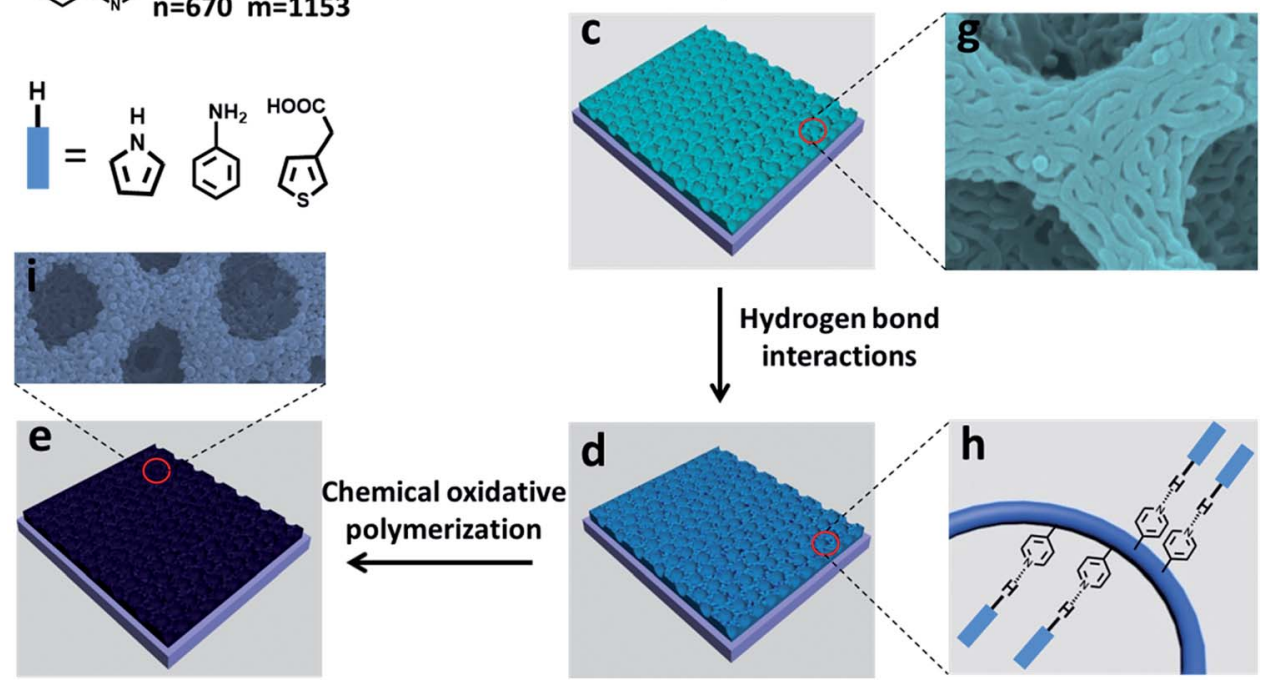

Scheme 1 Schematic illustration of fabricating stable porous polymer films by combining breath figures, microphase separation and swelling. (a) Polymer solution spread on cleaned glass substrate by drop-casting. (b) Honeycomb porous films after breath figures. (c) Hierarchically structured porous polymer films after swelling in ethanol. (d) Hydrogen bonding complexes formed after porous membranes immersed in pyrrole, aniline and 3-thiopheneacetic acid monomer solutions. (e) Nanostructured conducting polymer after chemical oxidative polymerization. (f), (g), (h) and (i) are corresponding magnified SEM images of (b), (c), (d) and (e), respectively. 
recrystallization three times from ethanol. Glass substrates were cut into $1 \times 1 \mathrm{~cm}^{2}$ and were cleaned prior to use in piranha solution (7:3 v/v of $\mathrm{H}_{2} \mathrm{SO}_{4} / \mathrm{H}_{2} \mathrm{O}_{2}$ ) and rinsed several times in deionized water and ethanol, then dried with nitrogen gas.

\section{Fabrication of hierarchically structured polymer films}

Polystyrene-block-poly(4-vinylpyridine) was synthesized by RAFT according to a reported procedure. ${ }^{11}$ Relative molecular weight and molecular weight distribution were determined by ${ }^{1} \mathrm{H}$ NMR and GPC, respectively. PS- $b$-P4VP synthesized in our work was dissolved in dry chloroform, using magnetic stirrer for several hours to yield $0.28 \mathrm{wt} \%$ polymer solutions. In a typical experiment, the polymer solutions were filtered with a $0.1 \mu \mathrm{m}$ Millipore membrane before use. The typical preparation procedure of breath figures process was carried out in a constant temperature and humidity chamber, and the relative humidity was monitored in real time by means of an internal humidity detector. A $30 \mu \mathrm{L}$ of the polymer solution was dropped on a clean glass substrate under $30 \%$ relative humidity at $25{ }^{\circ} \mathrm{C}$. Owing to the condensation of water vapor on the solution surface during the evaporation of chloroform, the transparent solution turned turbid. After complete evaporation of the solvent, the honeycomb ordered porous polymer films were removed from the chamber. Next, the self-assembly of the honeycomb ordered porous polymer films were carried out in ethanol bath at a given temperature with different time and nanopores were generated by swelling. Then put the hierarchically structured porous polymer films in vacuum drying oven at room temperature overnight.

\section{Fabrication of nanostructured conducting polymers}

The hierarchically structured porous polymer films prepared as above were immersed in $0.3 \mathrm{~mol} \mathrm{~L}^{-1}$ pyrrole ethanol solution in Petri dish and were allowed to soak for several hours at room temperature. Subsequently the films were washed with absolute ethyl alcohol for three times. Then the films were put into Petri dish containing saturated ferric chloride ethanol solution for several hours at room temperature. Finally the polypyrrole/ block copolymer (PPy/BCP) composite films were washed with absolute ethyl alcohol three times. PPy/BCP nanostructured conducting polymers were finally fabricated and dried under reduced pressure at $40{ }^{\circ} \mathrm{C}$. Polyaniline/block copolymer (PANI/ BCP) and poly(3-thiopheneacetic acid)/block copolymer (P3TAA/BCP) were prepared according to the similar steps. The concentration of aniline is $0.2 \mathrm{~mol} \mathrm{~L}^{-1}$ and the concentration of 3-thiopheneacetic acid is $0.04 \mathrm{~mol} \mathrm{~L}^{-1}$. PPy, PANI and P3TAA nanostructured conducting polymers could be obtained by were soaking the corresponding composites into dichloromethane to dissolve block copolymer.

\section{Fabrication of regular $\mathrm{SiO}_{2}$ honeycomb porous films}

The regular $\mathrm{SiO}_{2}$ honeycomb porous films were fabricated through the following processes. TEOS (0.52 $\mathrm{g}, 2.5 \mathrm{mmol})$ was dissolved in anhydrous ethanol $(5 \mathrm{~g})$, deionized water $(1.08 \mathrm{~g}, 60$ $\mathrm{mmol}$ ) was dropped into the above solution. After full mixing, hierarchically structured porous films were immersed in the mixed solution. After $48 \mathrm{~h}$, the films were took out and washed three times with ethanol. The silica/BCP composite films were soaked into dichloromethane solution to dissolve the block copolymer. Then the $\mathrm{SiO}_{2}$ honeycomb porous films were washed with dichloromethane three times, and dried in oven at $40{ }^{\circ} \mathrm{C}$.

\section{Characterization methods}

${ }^{1} \mathrm{H}$ NMR spectra were obtained using a JEOL JNM-ECA300 at 300 $\mathrm{MHz}$ with $\mathrm{CDCl}_{3}$. Gel permeation chromatography (GPC) was performed by a set of a Waters 515 HPLC pump. The surface morphologies of the fabricated films were observed using a field emission scanning electron microscopy (FE-SEM, $10 \mathrm{kV}$, SU8010) with energy dispersive X-ray spectrometry (EDX, $20 \mathrm{kV}$ ). Samples were pre-coated with platinum through an ion sputter prior to imaging. The ATR-FTIR spectra of the porous films were measured by Bruker tensor spectrophotometer. Before this spectral analysis, the film samples were dried in a vacuum oven at $80{ }^{\circ} \mathrm{C}$ overnight and stored in a vacuum desiccator. Electrochemical measurements were carried out on a CHI660B electrochemical workstation (CHI, Shanghai) in a standard one compartment three-electrode configuration cell with the platinum sheet as counter electrode, and saturated calomel electrode (SCE) as reference electrode in $1 \mathrm{~mol} \mathrm{~L}^{-1} \mathrm{KCl}$ solution. All the potentials mentioned later are versus SCE.

\section{Results and discussion}

\section{Fabrication of regular honeycomb porous films}

Four types of block copolymer, named as \#1 PS650- $b$-P4VP196, \#2 PS660- $b$-P4VP35, \#3 PS660- $b$-P4VP244, and \#4 PS650- $b$ P4VP1153, were synthesized by RAFT polymerization according to reported procedures. ${ }^{11}$ The imprint of water droplet on the membrane makes the films ordered honeycomb patterns. ${ }^{12,13}$ The ratio of $\mathrm{P} 4 \mathrm{VP} / \mathrm{PS}$ has big influence on the pore size and film morphology. ${ }^{\mathbf{1 4}}$ In Fig. 1, the diameter of the pore differs as the percentage of P4VP, presenting sizes of $1.68 \pm 0.3,0.45 \pm 0.4$, $0.88 \pm 0.1$, and $1.87 \pm 0.2 \mu \mathrm{m}$ for sample \#1 PS650- $b$-P4VP196, \#2 PS660- $b$-P4VP35, \#3 PS660- $b$-P4VP244, and \#4 PS650- $b$ P4VP1153, respectively. Sample \#1 and \#4 have the same PS block number, but P4VP block number of sample \#4 is doubled that of sample \#1. It can be found that the pore size of sample \#1 is bigger than sample \#4. Sample \#2 has the lowest P4VP/PS ratio among the four samples and the corresponding pore size is also smallest. Owing to the low content of P4VP in sample \#2, big size water droplets cannot be well stabilized and the obtained 2-dimentional patters are not very regular. However, the other three samples can stable relatively larger water droplets very well, and hence bigger pores with satisfactory regularity can be gotten.

\section{Fabrication of hierarchically structured polymer films}

Hierarchically structured polymer films can be fabricated by combining breath figures and microphase separation or other method. ${ }^{15,16}$ As shown in Scheme 1, PS- $b$-P4VP block copolymer porous films were used to fabricate hierarchically porous films after ethanol swelling. Fig. 2 demonstrates the generation of nanostructures of honeycomb porous films of different sample 

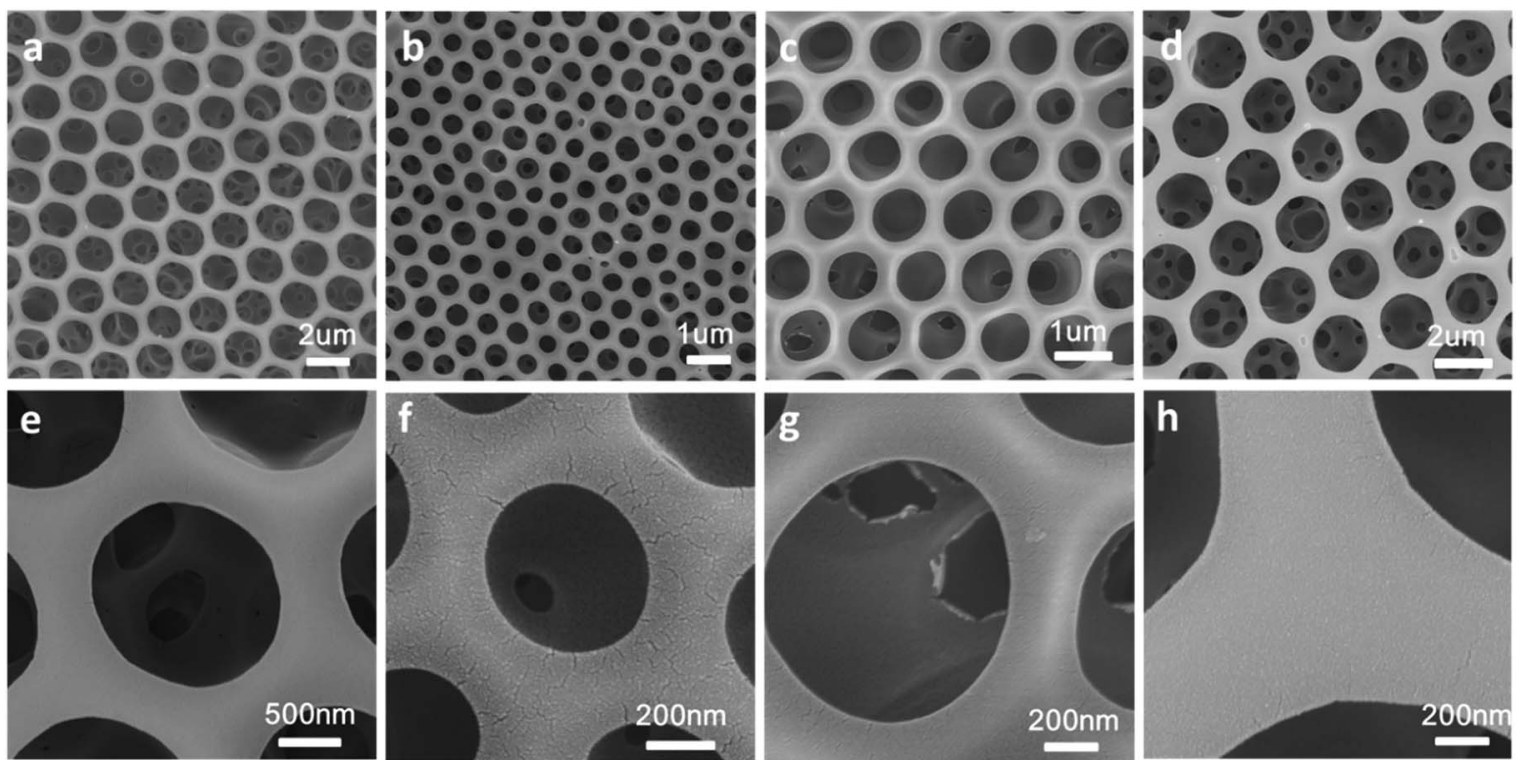

Fig. 1 SEM images of the honeycomb films fabricated by breath figures from sample \#1 PS650-b-P4VP196 (a); \#2 PS660-b-P4VP35 (b); \#3 PS660-b-P4VP244 (c); \#4 PS650-b-P4VP1153 (d). (e), (f), (g) and (h) are corresponding magnified SEM images of (a), (b), (c) and (d), respectively.

under $70^{\circ} \mathrm{C}$ in ethanol for 4 hours. The regularity of honeycomb films has little change after swelling. This is a very important step for the further improvement of stability. After swelling by ethanol, P4VP groups were thoroughly exposed and these hydrogen bonding sites distributed throughout the entire film. This gives the possibility for the stabilization of porous films. Otherwise, only the surface of the honeycomb film owns hydrogen bonding sites and the resultant structure is not stable after removing block copolymer. From Fig. 2e-h, we can clearly see that different samples show different microstructures at the wall. Worm-like bicontinuous nanostructures can be obviously seen in sample \#1, \#2 and \#3 while nanohole structures can be observed in sample \#4. This results can be explained by the phase diagram of a diblock copolymer with different volume fractions which has been reported by a lot of research. ${ }^{11}$ The four samples show different structures after swelling of amphiphilic block copolymer. The nanostructure in Fig. $2 \mathrm{~g}$ doesn't completely penetrate and the nanostructure in Fig. $2 \mathrm{~h}$ show nanopores. In Fig. 3, we compared the morphology of sample \#1 and \#2 which have similar PS blocks number, but significantly different block number of P4VP and we found the influence of P4VP block number is big. The nanochannel is
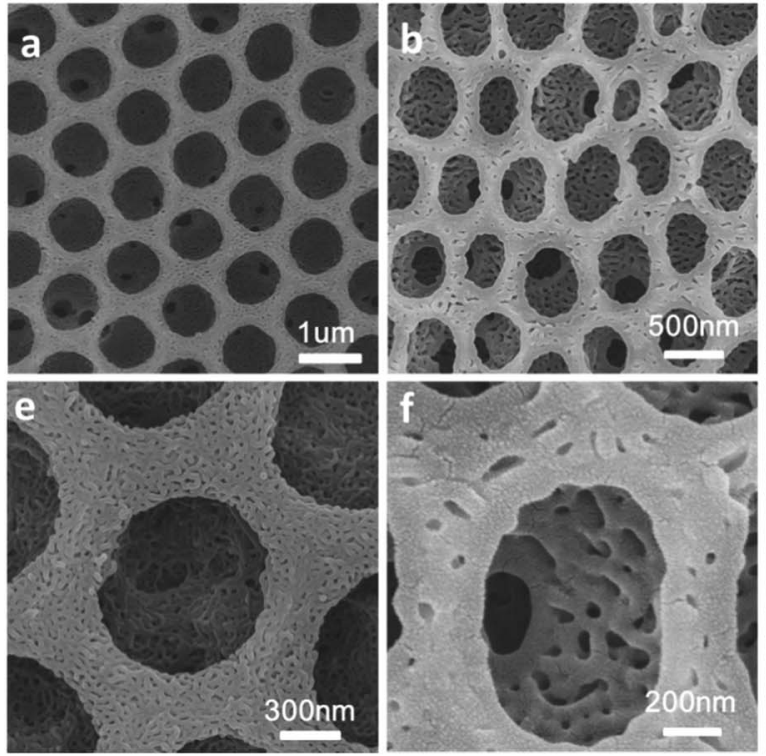
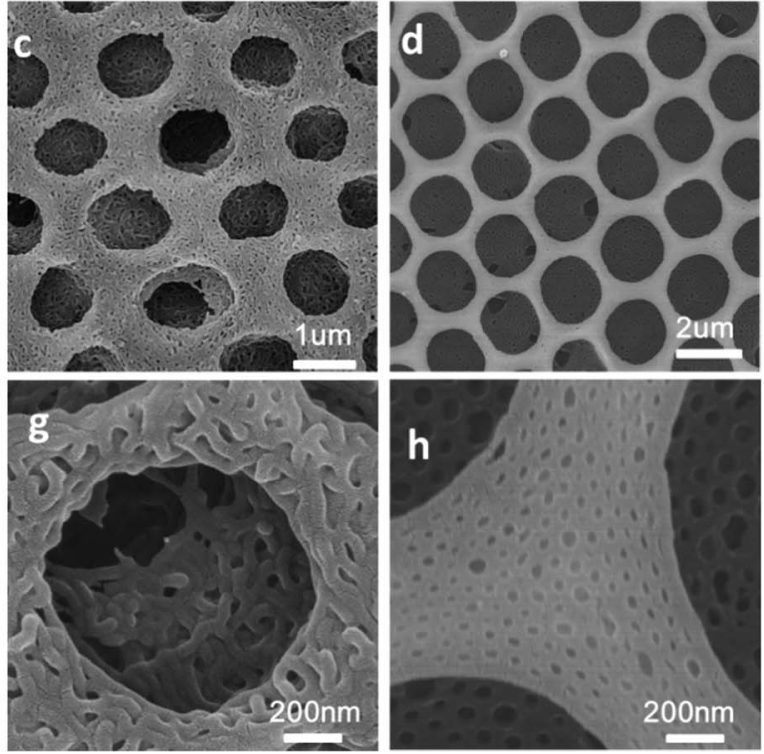

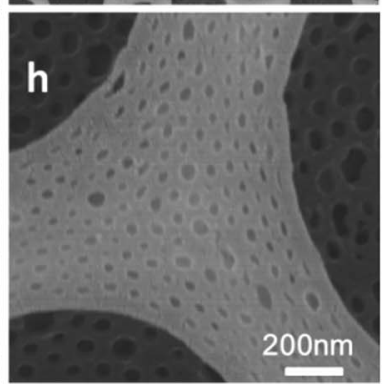

Fig. 2 SEM images of the hierarchically structured polymer films fabricated by breath figures, microphase separation and swelling from sample \#1 PS650-b-P4VP196 (a); \#2 PS660-b-P4VP35 (b); \#3 PS660-b-P4VP244 (c); \#4 PS650-b-P4VP1153 (d). (e), (f), (g) and (h) are corresponding magnified SEM images of (a), (b), (c) and (d), respectively. 

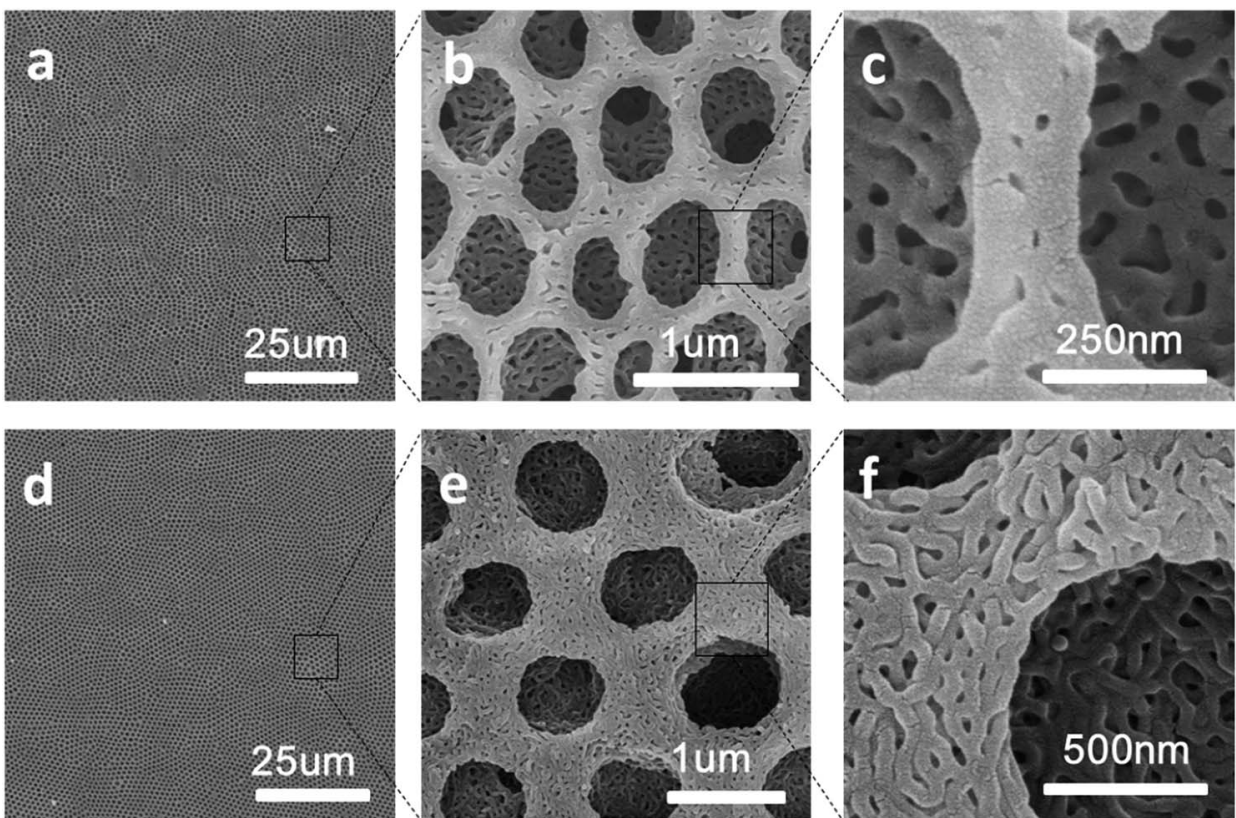

Fig. 3 SEM images in different magnification of the hierarchically structured polymer films fabricated by breath figures, microphase separation and swelling by ethanol from sample \#1 PS650-b-P4VP196 (a-c) and sample \#3 PS660-b-P4VP244 (d-f).

narrow for sample \#2 because it has a short P4VP block. When it was swelled in ethanol and volatilized in the air, the stretching and contraction of P4VP chain is not very obvious. Contrary to sample \#2, sample \#1 has the nanochannel structure. Besides the volume ratio of block copolymer, the morphology and size of the nanostructure can also be influenced by swelling time (Fig. 4). Sample \#1 is chosen to swell in ethanol for $1 \mathrm{~h}, 2 \mathrm{~h}, 3 \mathrm{~h}$ and $4 \mathrm{~h}$. From Fig. 4 , the nanochannel of the wall is becoming wider over time. One hour swelling time for the film is too short for the P4VP block to fully stretch. Therefore, the nanochannel shrinks and becomes narrow after the volatilization of ethanol. With the swelling time increasing, the chain extension is more and more complete, resulting a wider pore size at the wall. After $4 \mathrm{~h}$ swelling, the nanostructure of the film is best for the next step fabrication.

\section{Fabrication of nanostructured conducting polymers}

Hierarchically porous films are fabricated after breath figure and microphase separation as described above. Amphiphilic block copolymers (PS-b-P4VP) have been widely used for porous
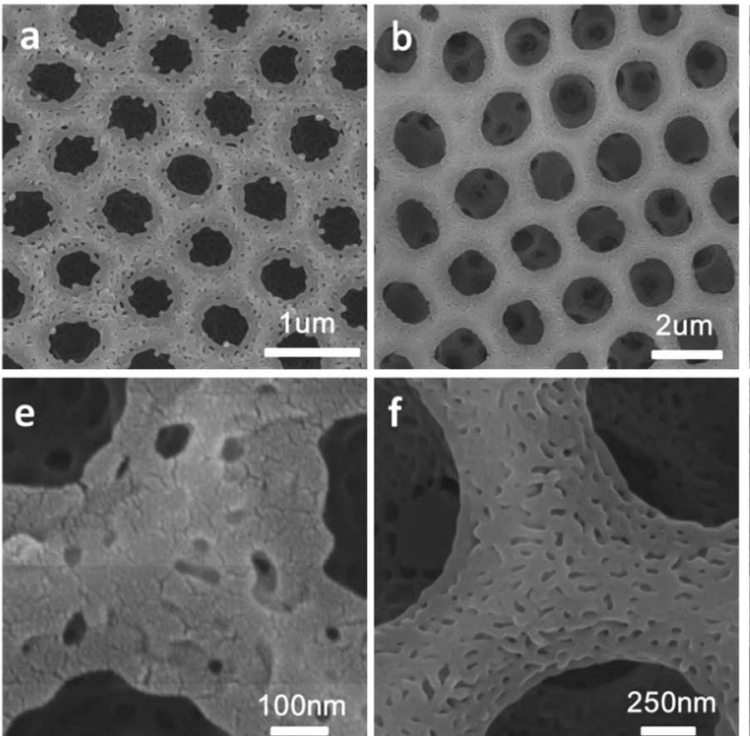
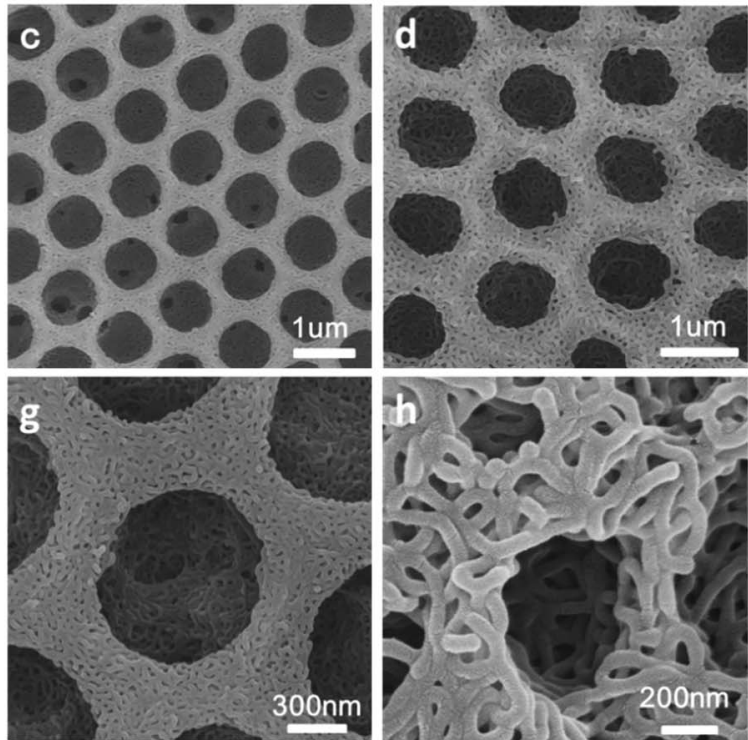

Fig. 4 SEM images of \#1 PS650-b-P4VP196 membranes swelling in ethanol at different time (a) 1 h; (b) 2 h; (c) 3 h; (d) 4 h. (e), (f), (g) and (h) are corresponding magnified SEM images of (a), (b), (c) and (d), respectively. 

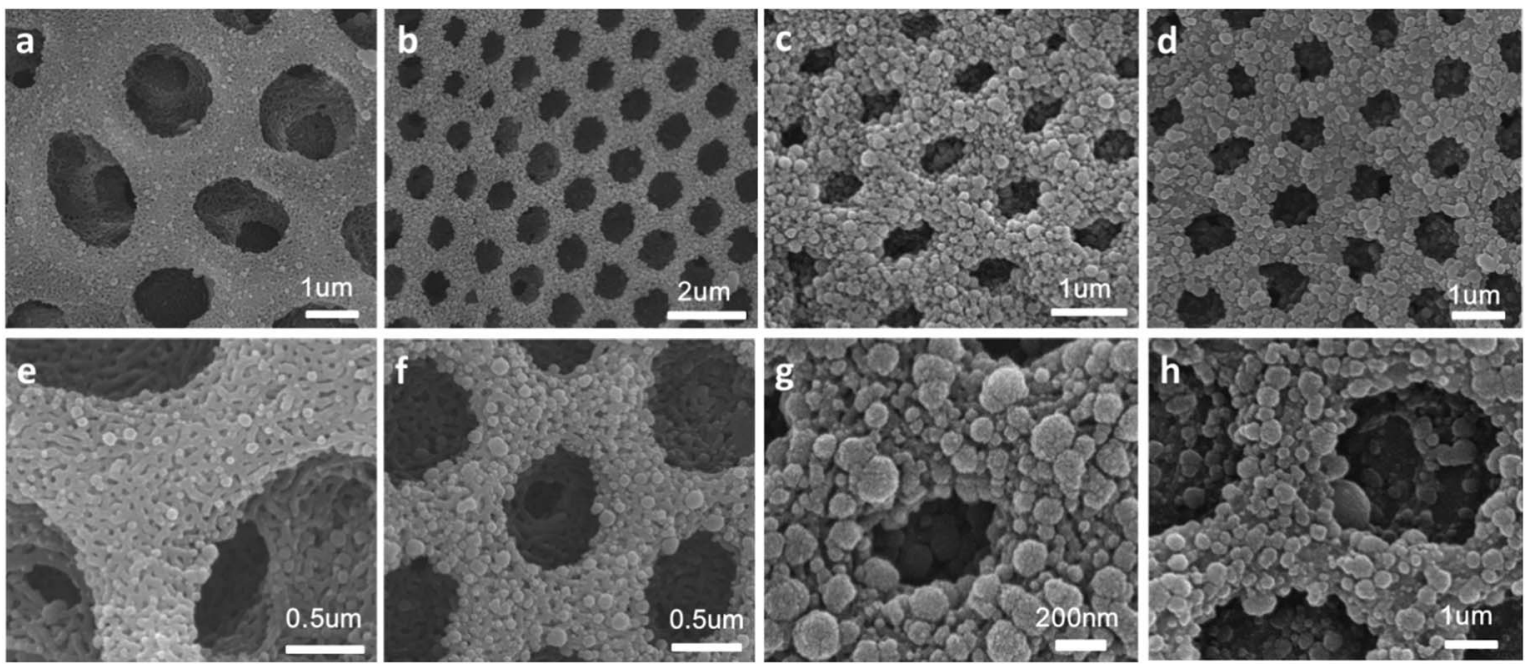

Fig. 5 SEM images of nanostructure conducting polymer composites (PPy/BCP) polymerized at (a) 12 h, (b) 24 h and (c) 48 h. (d) Nanostructure conducting polymer (PPy) after removing BCP from PPy/BCP. (e), (f), (g) and (h) are corresponding magnified SEM images of (a), (b), (c) and (d), respectively.

template owing to the contained P4VP moieties which can form hydrogen bonds complex with other monomers containing hydrogen bond donor such as $-\mathrm{COOH},-\mathrm{NH}_{2},-\mathrm{NH}-$. After the microphase separation process and swelling by ethanol, 4-vinyl pyridine moieties as hydrogen bond acceptors are completely exposed throughout the films. Many researchers have done a lot of research in this area. ${ }^{17-24}$ In this work, we choose pyrrole, aniline and 3-thiopheneacetic acid as the hydrogen bond donor because they are easily polymerized by chemical oxidative polymerization and these polymers are solvent and thermal stable. After polymerization, black polypyrrole is achieved. Fig. 5a-c are SEM images of polypyrrole ( $\mathrm{PPy} / \mathrm{BCP})$ at polymerization time $12 \mathrm{~h}, 24 \mathrm{~h}$ and $48 \mathrm{~h}$. It can be seen that with the increasing polymerization time, the surface of the film become more and more rough, indicating the increase of the polypyrrole film thickness. In Fig. 5e, small particles are observed and the wormlike structure is thin. In Fig. $5 \mathrm{f}$ polypyrrole particles formed on the film, leading to increased pore wall thickness. Fig. 5g is PPy/ BCP polymerized for $48 \mathrm{~h}$, the polypyrrole particles are quiet obvious and the diameter of honeycomb pore becomes very small. The polypyrrole almost fill the wormlike pores. Interconnected nanostructured conducting composites were immersed into dichloromethane for 24 hours to remove the block copolymer and dried in the oven at $80{ }^{\circ} \mathrm{C}$ overnight. The structure of honeycomb porous films fabricated by BF keeps well in solvent (Fig. 5d). This means that the honeycomb porous films
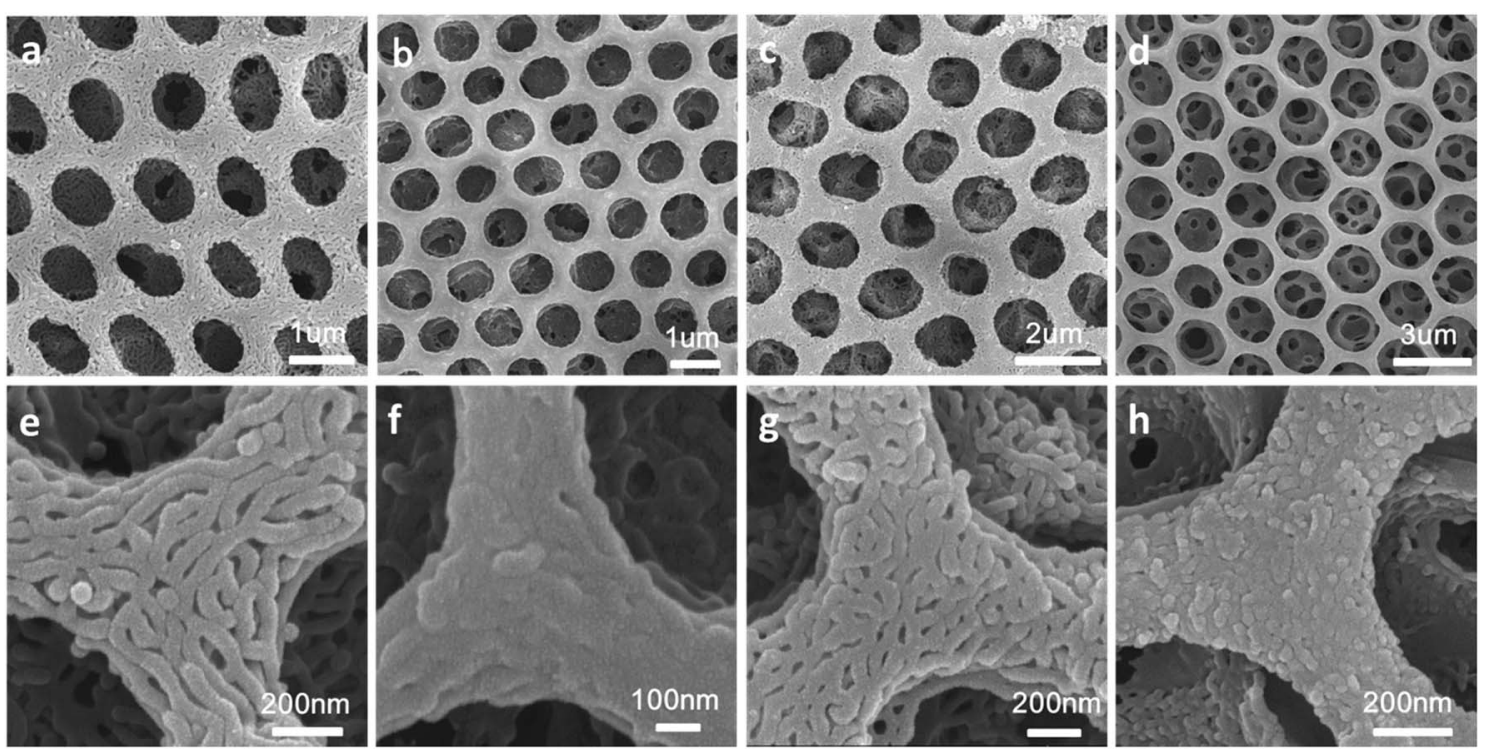

Fig. 6 (a) and (c) SEM images of nanostructure conducting polymer composites (PANI/BCP and P3TAA/BCP) polymerized at $48 \mathrm{~h}$. (b) and (d) SEM images of nanostructure conducting polymer (PANI and P3TAA) after removing BCP from PANI/BCP and P3TAA/BCP. (e), (f), (g) and (h) are corresponding magnified SEM images of (a), (b), (c) and (d), respectively. 

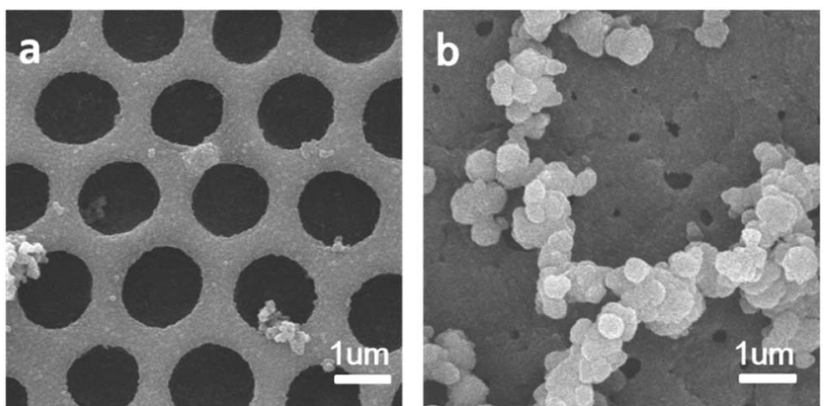

Fig. 7 (a) SEM images of nanostructure conducting polymer composites (PPy/BCP), honeycomb films were not swelling in ethanol. (b) SEM images of nanostructure conducting polymer (PPy) after removing $B C P$ from $P P y / B C P$.

can be well stabilized by PPy. Moreover, we choose aniline and 3thiopheneacetic acid as the monomer too. After polymerization for $48 \mathrm{~h}$, the colour of polyaniline composites (PANI/BCP) and poly(3-thiopheneacetic acid) composites (P3TAA/BCP) are dark green and red brown. Fig. 6a and $\mathrm{c}$ is PANI/BCP and P3TAA/BCP, and Fig. 6e and $\mathrm{g}$ is magnitude images of Fig. $6 \mathrm{a}$ and c. The surfaces of PANI/BCP and P3TAA/BCP are smoother than that of PPy/BCP since PANI and P3TAA particles is almost invisible on the surface. Fig. $6 \mathrm{~b}$ and $\mathrm{d}$ is the obtained PANI and P3TAA after the BCP dissolved in dichloromethane from their corresponding composites. From Fig. $6 f$ and $h$, the magnified images of $b$ and $d$, we can see that the structure of honeycomb porous films fabricated by BF still keeps well. In order to conclude that hydrogen bonding sites distributed throughout the entire film, we chose honeycomb films without swelling as a contrast. Honeycomb films are obvious destroyed after remove BCP from $\mathrm{PPy} / \mathrm{BCP}$ nanocomposite (Fig. $7 \mathrm{~b}$ and c). It is proved that the hydrogen bonds are distributed on the entire film after the swelling of the honeycomb films, so the honeycomb structure can be maintained well when the block copolymer is removed.

Further exploring the thermal and solvent stability of the obtained honeycomb films were carried out. The thermal resistance of the honeycomb films of PPy, PANI and P3TAA is performed by heating the films up to $200^{\circ} \mathrm{C}$ on a hot stage in air with a programmed temperature. SEM is used to assess the 3dimensional structure. It is found that the honeycomb
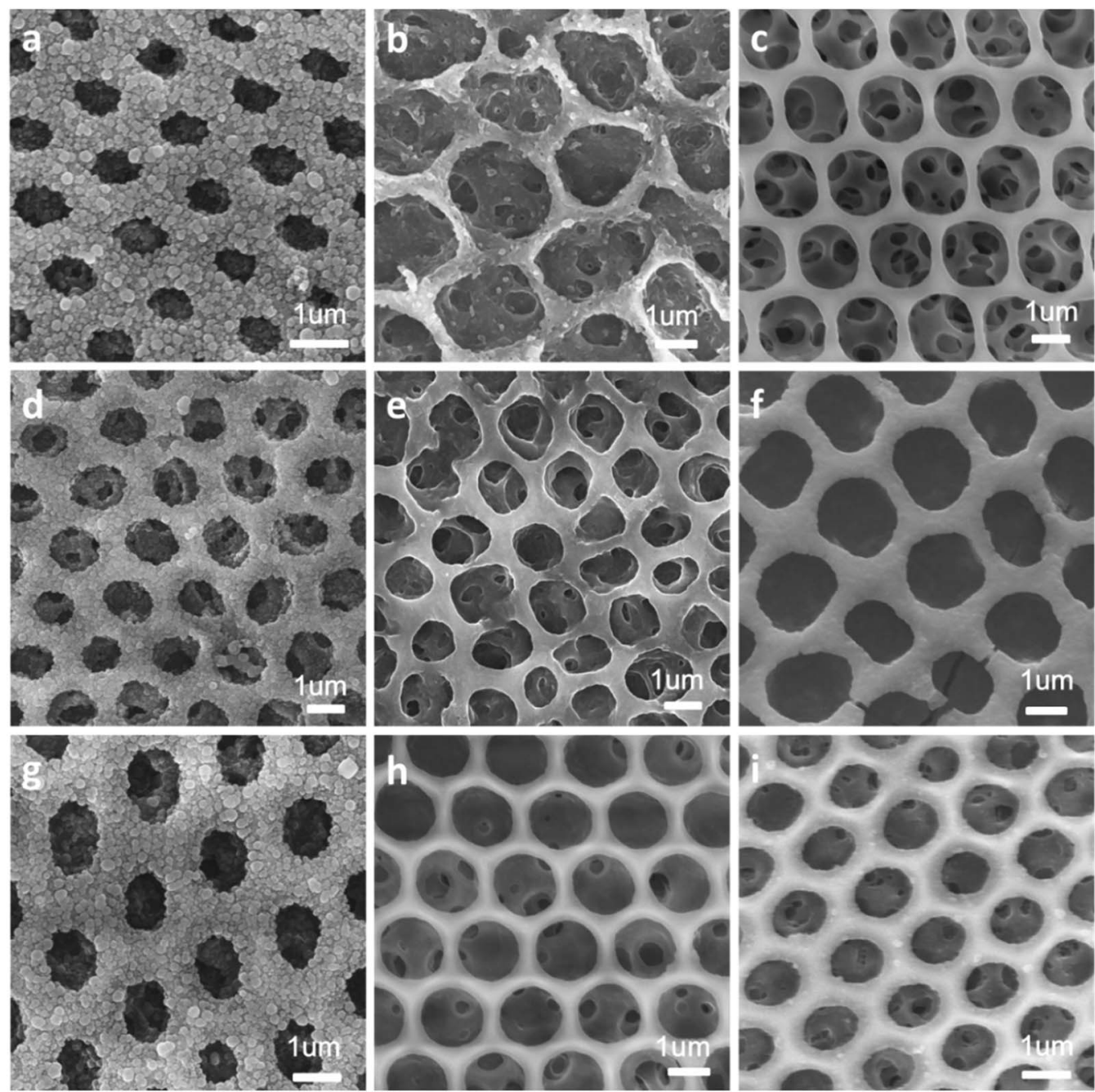

Fig. 8 SEM images of nanostructure conducting polymers of PPy, PANI and P3TAA respectively after put into different conditions. (a)-(c) at $200{ }^{\circ} \mathrm{C}$ in oven. (d) - (f) soaking in THF. (g)-(i) soaking in DMF. 
structures of PPy and P3TAA (Fig. 8a and c) keep well under such high temperature. These 3-dimensional structures keep stable too. Honeycomb film of PANI has a slight deformation, but the honeycomb structure remained intact (Fig. 8b). Additionally, the honeycomb films in our work are not only thermal stable, but also organic solvent stable. Immersed in THF and DMF which are good solvents for most polymers, honeycomb films of PPy, PANI and P3TAA are still stable (Fig. 8d-i). To further proving solvent stability of nanomaterials, we put honeycomb films in hydrochloric solution $(\mathrm{pH}=2)$, sodium hydroxide solution $(\mathrm{pH}=12)$ and saturated sodium porous chloride solution to explore their stabilities (Fig. 9). The honeycomb structures keeps well in them. According to the results, we conclude that the honeycomb film is stable in a wide variety of organic solvents, including chloroform, toluene, $\mathrm{CS}_{2}$ and benzene ${ }^{8 \boldsymbol{d}}$ and inorganic solvents, including acid, alkaline and salt solution. In this article, as a proof of concept, only three monomers as hydrogen bond donors are chosen. Nevertheless according to the principle for preparing stable structure, there are a large number of monomers can be selected for the above purpose.

\section{ATR-FTIR spectrum characterization}

ATR-FTIR analysis was done to auxiliary prove the successful polymerization of pyrrole, aniline and 3-thiopheneacetic acid (Fig. 10). Results shown in Fig. 8 indicate the presence of characteristic peaks of PPy at 1584, 1470, 1040, 930-840 $\mathrm{cm}^{-1}$

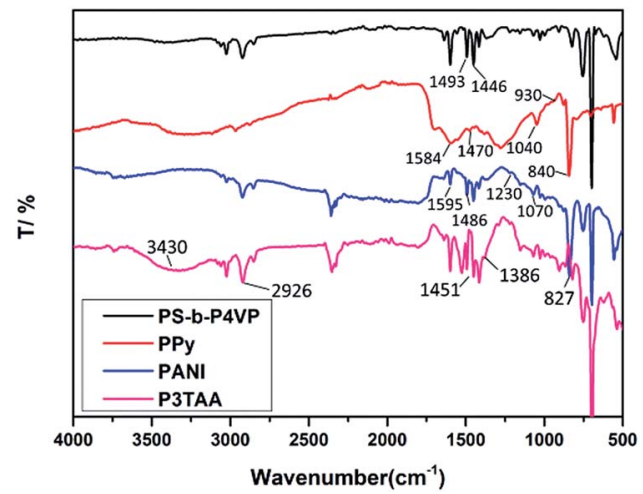

Fig. 10 ATR-FTIR spectrum of PS-b-P4VP, PPy, PANI and P3TAA.
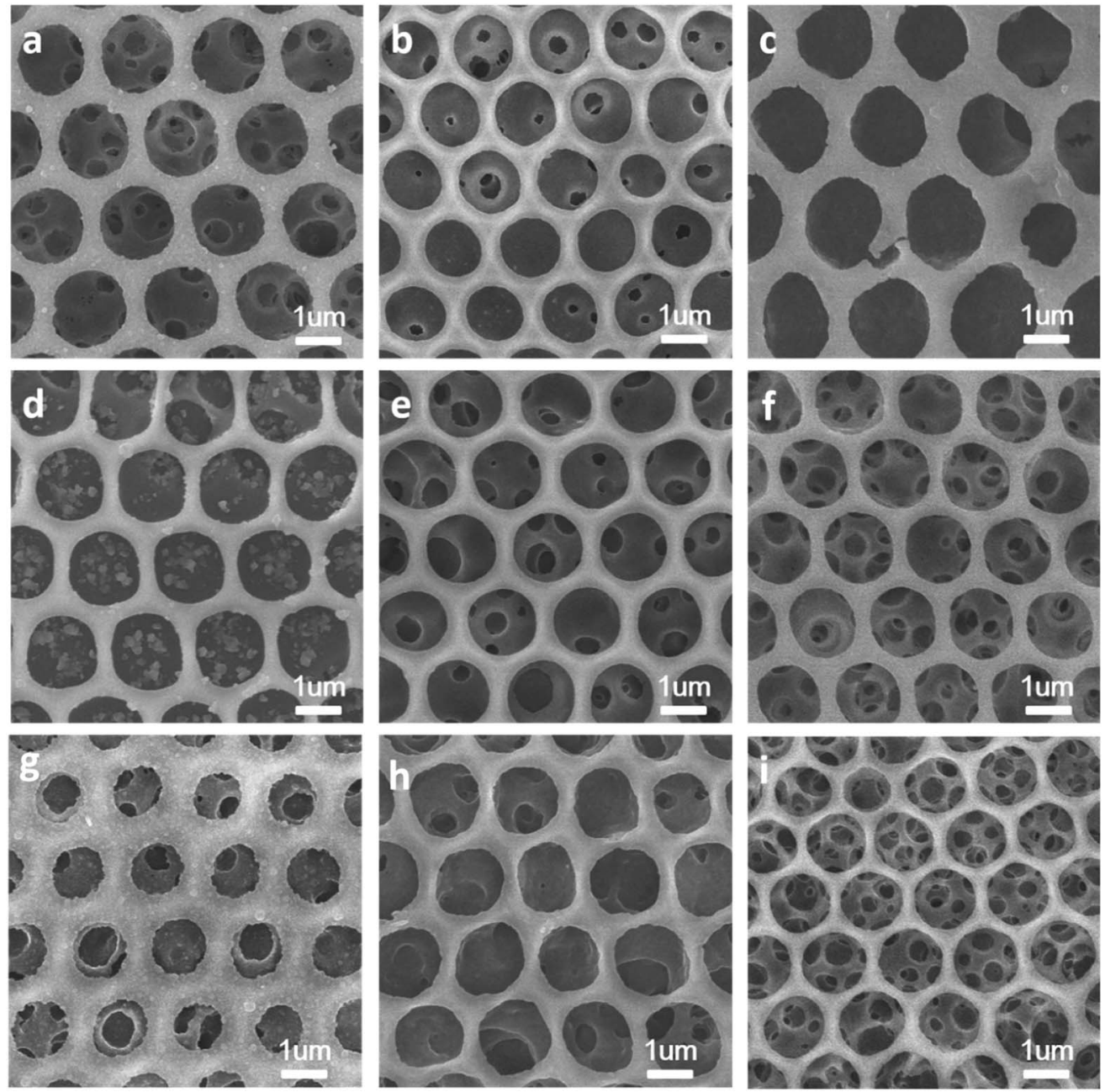

Fig. 9 SEM images of nanostructure conducting polymers of PPy, PANI and P3TAA respectively after put into in different solvents. (a) -(c) soaking in hydrochloric solution $(\mathrm{pH}=2),(\mathrm{d})$-(f) soaking in sodium hydroxide solution $(\mathrm{pH}=12)$ and $(\mathrm{g})$-(i) saturated sodium chloride solution. 
attributed to the pyrrole ring stretching, conjugated $\mathrm{C}-\mathrm{N}$ stretching, $\mathrm{C}-\mathrm{H}$ stretching vibration, and $\mathrm{C}-\mathrm{H}$ deformation, respectively. While the characteristic peaks of PANI are 1595, 1486, 1230, 1070 $\mathrm{cm}^{-1}$ which correspond to quinine and benzene stretching ring deformation and $\mathrm{C}-\mathrm{N}$ and $\mathrm{N}=\mathrm{Q}=\mathrm{N}$ ( $\mathrm{Q}$ denotes the quinoid rings). The characteristic peaks of P3TAA at 3430, 2926, 1627, 1451 and $1386 \mathrm{~cm}^{-1}$ which were attributed to the vibrational stretching of $-\mathrm{OH}$ in the $-\mathrm{COOH}$, stretching vibration peak of $-\mathrm{C}-\mathrm{H}-$ in thiophene ring, stretching vibration peak of $-\mathrm{C}=\mathrm{O}$, stretching vibration peak of in thiophene ring and stretching vibration peak of $-\mathrm{C}=\mathrm{C}-$ in thiophene ring. These peak positions are well matched with the reported peaks for PPy, PANI and P3TAA. ${ }^{25-27}$

\section{Electrochemical characterization}

Cyclic voltammetry (CV) is used to investigate the conductivity and proved the polymerization of PPy, PANI and P3TAA. The CV tests are carried out from -1.0 to $-0.5 \mathrm{~V}$ for PPy and BCP blank films, with the scan rates of $100 \mathrm{mV} \mathrm{s}^{-1}$. The electrolyte is $1 \mathrm{~mol}$ $\mathrm{L}^{-1} \mathrm{KCl}$ solution. Fig. 11a presents the CV curves which were obtained $10^{\text {th }}$ cycle to ensure the PPy films activated and stable. We observed that the maximum faradic current of PPy is large and the shape of CV curves for PPy is rectangle-like, which is the characteristic of electrochemical capacitors. This shape is well matched with the reported peaks for PPy. ${ }^{28}$ The CV tests are carried out from -0.2 to $0.8 \mathrm{~V}$ for PANI and P3TAA films, with
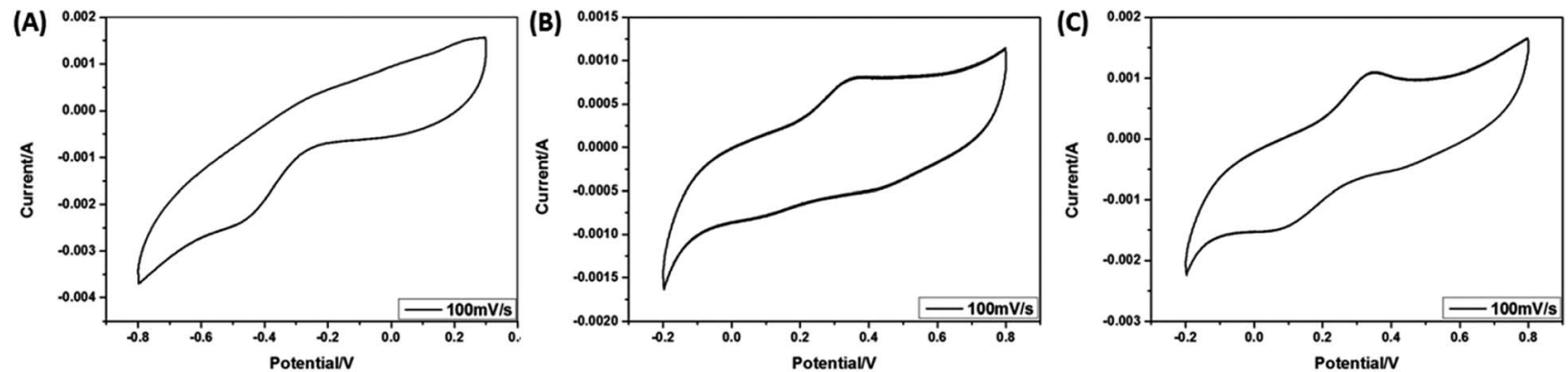

Fig. 11 (A) Cyclic voltammograms curves of PPy. (B) Cyclic voltammograms of PANI. (C) Cyclic voltammograms of P3TAA.
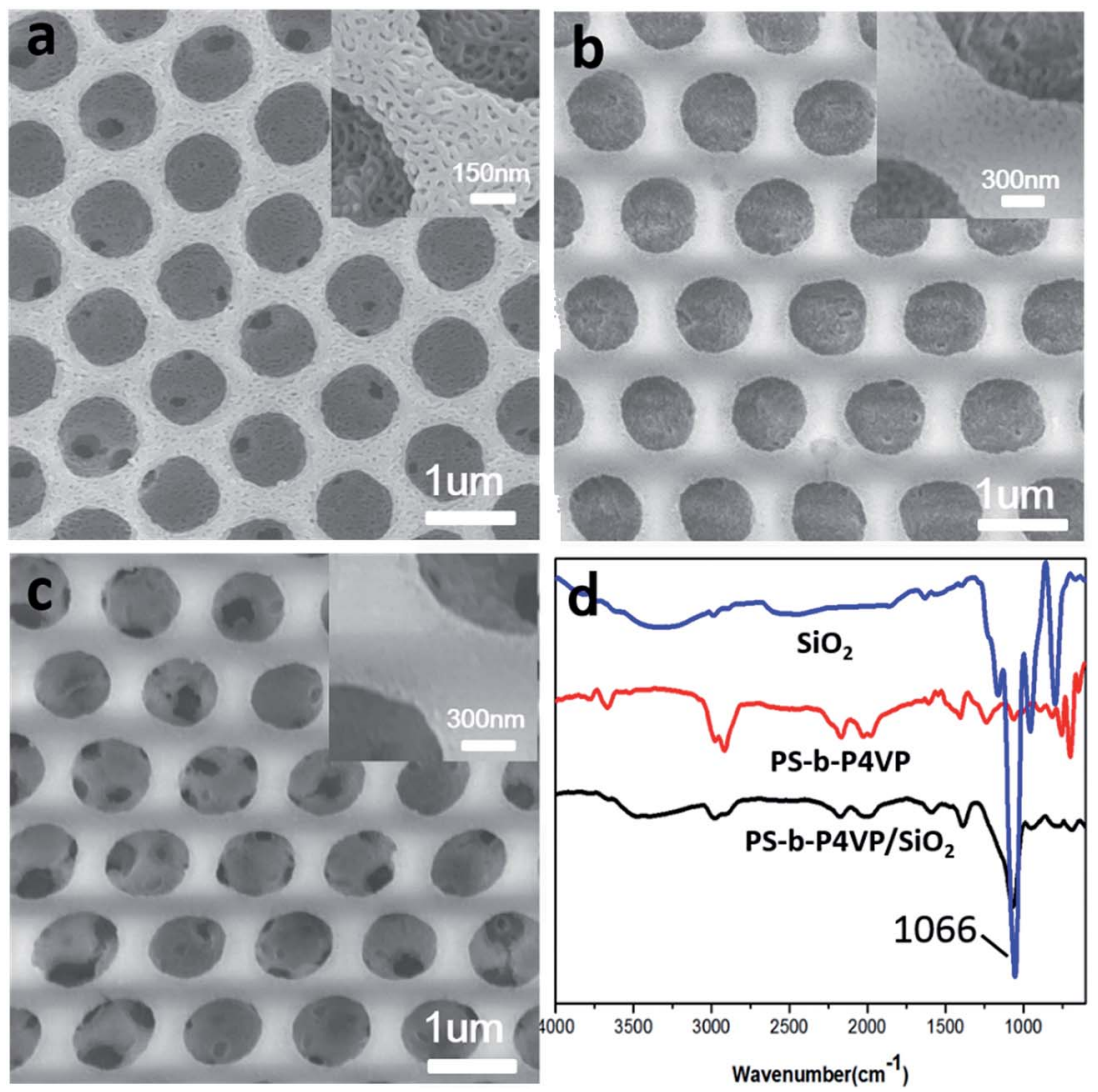

Fig. 12 (a) SEM images of the hierarchically structured polymer films fabricated by breath figures and micro-phase separation by ethanol from 1\#PS650-b-P4VP196. (b) SEM images of $\mathrm{SiO}_{2} / \mathrm{BCP}$ composite films. (c) SEM images of regular $\mathrm{SiO}_{2}$ honeycomb porous films. (d) ATR-FTIR spectrum of PS- $b-\mathrm{P} 4 \mathrm{VP}, \mathrm{SiO}_{2} / \mathrm{BCP}$ composite films and $\mathrm{SiO}_{2}$ honeycomb films. 
the scan rates of $100 \mathrm{mV} \mathrm{s}^{-1}$ containing $1 \mathrm{~mol} \mathrm{~L}^{-1} \mathrm{H}_{2} \mathrm{SO}_{4}$ solution as electrolyte. Fig. $11 \mathrm{~b}$ and $\mathrm{c}$ are $\mathrm{CV}$ of PANI and P3TAA respectively showing good electrochemical conductivity. The shape of CV curves of PANI and P3TAA are well matched with the reported peaks. ${ }^{29-31}$

\section{Fabrication of regular $\mathrm{SiO}_{2}$ honeycomb porous films}

The regular $\mathrm{SiO}_{2}$ honeycomb porous films were gotten in our work (Fig. 12c). Attributed to the swelling by ethanol, all the 4VP groups were expose solvent to remove BCP. Afterwards, regular $\mathrm{SiO}_{2}$ honeycomb porous films were obtained (Fig. 12c). The honeycomb structure kept very well. This is a very simple and extensible method to stable honeycomb porous structure. To verify the hydrolysis of TEOS, ATR-FITR was used to characterize PS- $b$-P4VP honeycomb films, $\mathrm{SiO}_{2} / \mathrm{BCP}$ composite films and $\mathrm{SiO}_{2}$ honeycomb films. As shown in Fig. 12d, the absorption peak PS- $b$-P4VP film was the aromatic ring on styrene and pyridine groups. For the $\mathrm{SiO}_{2} / \mathrm{BCP}$ composite films and $\mathrm{SiO}_{2}$ honeycomb films, the $1066 \mathrm{~cm}^{-1}$ peak was attributed to the characteristic peak of $\mathrm{Si}-\mathrm{O}-\mathrm{Si}$ asymmetric stretching vibration. The wide absorption band at $3000 \mathrm{~cm}^{-1}$ to $3600 \mathrm{~cm}^{-1}$ was the characteristic peaks of $-\mathrm{OH}$ absorption peak of $\mathrm{Si}-\mathrm{OH}$. The 960 $\mathrm{cm}^{-1}, 784 \mathrm{~cm}^{-1}$ peak was the characteristic peak of $\mathrm{SiO}_{2}$ tetrahedron and $\mathrm{Si}-\mathrm{O}-\mathrm{Si}$ symmetric stretching vibration. ${ }^{32}$

\section{Conclusions}

In order to realize the potential application of breath figure films, various strategies have been presented to improve the stability of these films in harsh environment. However, more effective methods to stable the porous films by BFs are still highly desirable, especially for developing a versatile platform to fulfil this goal. Herein, by combined use of breath figures process and microphase separation of PS- $b$-P4VP, a general method has been proposed by us to produce stable porous nanomaterials. Owing to the fantastic feature of the exposed pyridine moieties, its vitally significant roles as hydrogen bond acceptor or alkaline catalyst were utilized to form various hydrogen bond complexes or inorganic particles on the skeleton of a widely used block polymer PS- $b$-P4VP, for the further stabilizing the porous films. Notably, any organic molecule containing hydrogen bond donor or inorganic precursor with the ability to be catalytically hydrolyzed by pyridine moieties can be used to stable the BF films. Thus, the proposed method by us paves a versatile and effective way to prominently enhance the stability of the BF resultant hierarchically structured porous polymer films, clearly highlighting the significant value and enormous potential for the further applications.

\section{Acknowledgements}

This work was financially supported by National Science Foundation of China (No. 20141300592) and the Program (KY201402012) on Demonstration and Capacity Building of Drinking Water Treatment in East-Africa by the Ministry of Science and Technology of China (MOST).

\section{Notes and references}

1 G. Widawski, M. Rawiso and B. François, Nature, 1994, 369, 387.

2 (a) N. Maruyama, T. Koito, J. Nishida, T. Sawadaishi, X. Cieren, K. Ijiro, O. Karthaus and M. Shimomura, Thin Solid Films, 1998, 327-329, 854-856; (b) N. Maruyama, O. Karthaus, K. Ijiro, M. Shimomura, T. Koito, S. Nishimura, T. Sawadaishi, N. Nishi and S. Tokura, Supramol. Sci., 1998, 5, 331-336; (c) Y. Tian, Q. Jiao, H. Ding, Y. Shi and B. Liu, Polymer, 2006, 47, 3866-3873; (d) M. H. Stenzel, T. P. Davis and A. G. Fane, J. Mater. Chem., 2003, 13, 2090-2097; (e) H. M. Ma and J. C. Hao, Chem. Soc. Rev., 2011, 40, 5457-5471.

3 A. J. Zhang, H. Bai and L. Li, Chem. Rev., 2015, 115, 98019868.

4 (a) C. Liu, C. Gao and D. Yan, Angew. Chem., Int. Ed., 2007, 46, 4128-4131; (b) D. Ishii, H. Yabu and M. Shimomura, Colloids Surf., A, 2008, 313-314, 590-594; (c) B. Englert, S. Scholz, J. P. Leech, M. Srinivasarao and U. H. F. Bunz, Chem.-Eur. J., 2005, 11, 995-1000.

5 (a) L. S. Wan, J. W. Li, B. B. Ke and Z. K. Xu, J. Am. Chem. Soc., 2012, 134, 95-98; (b) Y. Ou, C. Lv, W. Yu, Z. Mao, L. Wan and Z. Xu, ACS Appl. Mater. Interfaces, 2014, 6, 22400-22407; (c) C. Du, A. J. Zhang, H. Bai and L. Li, ACS Macro Lett., 2013, 2, 27-30.

6 (a) X. C. Sun, C. Bruckner, M. P. Nieh and Y. Lei, ACS Macro Lett., 2014, 2, 14613-14621; (b) C. Wang, Q. Liu, X. Shao, G. Yang, H. Xue and X. Hu, Talanta, 2007, 71, 178-185.

7 (a) S. Chen, X. Lu, Z. Huang and Q. Lu, Chem. Commun., 2015, 51, 5698-5701; (b) H. J. Lee, C. S. Lee and K. Y. Cho, ACS Appl. Mater. Interfaces, 2014, 6, 16493-16497; (c) L. S. Wan, Q. L. Li, P. C. Chen and Z. K. Xu, Chem. Commun., 2012, 48, 4417-4419.

8 (a) A. Bolognesi, F. Galeotti, J. Moreau, U. Giovanella, W. Porzio, G. Scavia and F. Bertini, J. Mater. Chem., 2010, 20, 1483-1488; (b) T. Kabuto, Y. Hashimoto and O. Karthaus, Adv. Funct. Mater., 2007, 17, 3569-3573; (c) L. Li, Y. W. Zhong, J. L. Gong, J. Li, J. A. Li, J. Huang and Z. Ma, J. Colloid Interface Sci., 2011, 354, 758-764; (d) L. Li, C. Chen, J. Li, A. Zhang, X. Liu, B. Xu, S. Gao, G. Jin and Z. Ma, J. Mater. Chem., 2009, 19, 2789-2796; (e) O. Karthaus, Y. Hashimoto, K. Kon and Y. Tsuriga, Macromol. Rapid Commun., 2007, 28, 962-965; (f) Y. Nakamichi, Y. Hirai, H. Yabu and M. Shimomura, J. Mater. Chem., 2011, 21, 3884-3889.

9 B. Erdogan, L. L. Song, J. N. Wilson, J. O. Park, M. Srinivasarao and U. H. F. Bunz, J. Am. Chem. Soc., 2004, 126, 3678-3679.

10 (a) L. Li, Y. W. Zhong, C. Y. Ma, J. Li, C. K. Chen, A. J. Zhang, D. L. Tang, S. Y. Xie and Z. Ma, Chem. Mater., 2009, 21, 49774983; (b) J. L. Gong, L. C. Sun, Y. W. Zhong, Y. C. Ma, L. Li, S. Y. Xie and V. Svrcek, Nanoscale, 2012, 4, 278-283; (c) C. Y. Ma, Y. W. Zhong, J. Li, C. K. Chen, J. L. Gong, S. Y. Xie, L. Li and Z. Ma, Chem. Mater., 2010, 22, 2367-2374. 
11 (a) B. Q. Zhang, G. D. Chen, C. Y. Pan, B. Luan and C. Y. Hong, J. Appl. Polym. Sci., 2006, 102, 1950-1958; (b) J. J. Haven, C. G. Sanchez, D. J. Keddie, G. Moad, S. H. Thang and U. S. Schubert, Polym. Chem., 2014, 5, 5236-5246; (c) M. M. Bloksma, S. Hoeppener, C. Dhaese, K. Kempe, U. Mansfeld, R. M. Paulus, J. F. Gohy, U. S. Schubert and R. Hoogenboom, Soft Matter, 2012, 8, 165-172.

12 H. T. Lord, J. F. Quinn, S. D. Angus, M. R. Whittaker, M. H. Stenzel and T. P. Davis, J. Mater. Chem., 2003, 13, 2819-2824.

13 K. H. Wong, T. P. Davis, C. Barner-kowollik and M. H. Stenzel, Polymer, 2007, 48, 4950-4965.

14 (a) A. J. Zhang, H. Bai and L. Li, Chem. Rev., 2015, 115, 98019868; (b) P. Escale, L. Rubatat, L. Billon and M. Save, Eur. Polym. J., 2012, 48, 1001-1025.

15 (a) C. Cummins, T. Ghoshal, J. D. Holmes and M. A. Morris, Adv. Mater., 2016, 28, 5586-5618; (b) P. Escalé, M. Save, A. Lapp, L. Rubatat and L. Billon, Soft Matter, 2010, 6, 3202-3210.

16 (a) Z. Zhang, X. J. Hao, P. A. Gurr, A. Blencowe, T. C. Hughes and G. G. Qiao, Aust. J. Chem., 2012, 65, 1186-1190; (b) H. M. Ma and J. C. Hao, Chem.-Eur. J., 2010, 16, 655-660; (c) D. W. Fan, X. F. Jia, P. Q. Tang, J. C. Hao and T. B. Liu, Angew. Chem., 2007, 119, 3406-3409; (d) D. W. Fan, X. F. Jia, P. Q. Tang, J. C. Hao and T. B. Liu, Angew. Chem., Int. Ed., 2007, 46, 3342-3345.

17 (a) D. Beattie, K. H. Wong, C. Williams, L. A. Poole-Warren, T. P. Davis, C. Barner-Kowollik and M. H. Stenzel, Biomacromolecules, 2006, 7, 1072-1082; (b) Y. Q. Han, Q. Zhang, F. L. Han, C. X. Li, J. F. Sun and Y. Lu, Polymer, 2012, 53, 2599-2603.

18 F. Galeotti, A. Andiscova, S. Yunus and C. Botta, Soft Matter, 2012, 8, 4815-4821.

19 (a) W. V. Zoelen, S. Bondzic, T. F. Landaluce, J. Brondijk, K. Loos, A. J. Schouten, P. Rudolf and G. Brinke, Polymer, 2009, 50, 3617-3625; (b) J. F. Zang, C. M. Li, S. J. Bao, X. Q. Cui, Q. L. Bao and C. Q. Sun, Macromolecules, 2008, 41, 7053-7705; (c) A. Fakhry, F. Pillier and C. DebiemmeChouvy, ACS Macro Lett., 2014, 2, 9859-9865; (d) Y. Hou, L. Y. Chen, L. Zhang, J. L. Kang, T. Fujita, J. H. Jiang and M. W. Chen, J. Power Sources, 2013, 225, 304-310; (e) K. B. Xu, X. J. Huang, Q. Liu, R. Zou, W. Li, X. J. Liu, S. J. Li, J. M. Yang and J. Q. Hu, ACS Macro Lett., 2014, 2, 16731-16739.
20 (a) B. K. Kuila, B. Nandan, M. Bohme, A. Janke and M. Stamm, Chem. Commun., 2009, 5749-5751; (b) B. K. Kuila and M. Stamm, J. Mater. Chem., 2010, 20, 60866094.

21 D. Y. Ma, G. Y. Shi, H. Z. Wang, Q. H. Zhang and Y. G. Li, ACS Macro Lett., 2014, 2, 13541-13549.

22 (a) C. L. Yu, J. Zhai, Z. Li, M. X. Wan, M. Y. Gao and L. Jiang, Thin Solid Films, 2008, 516, 5107-5110; (b) H. D. Tran, J. M. D'Arcy, Y. Wang, P. J. Beltramo, V. A. Strong and R. B. Kaner, J. Mater. Chem., 2011, 21, 3534-3550.

23 (a) Y. Lu, Y. Ren, L. Wang, X. D. Wang and C. X. Li, Polymer, 2009, 50, 2035-2039; (b) Y. T. Tan, F. Ran, L. R. Wang, L. B. Kong and L. Kong, J. Appl. Polym. Sci., 2013, 127, 1544-1549.

24 (a) D. Bhattacharyya, R. Yang and K. K. Gleason, J. Mater. Chem., 2012, 22, 17147-17152; (b) P. K. Routh, D. Nykypanchuk, T. A. Venkatesh and M. Cotlet, Adv. Funct. Mater., 2015, 25, 5902-5909; (c) Y. C. Wang, S. Y. Tao, Y. L. An, S. Wu and C. G. Meng, ACS Macro Lett., 2013, 1, 8876-8887; (d) C. Janakya, B. Endrodia, K. Kovacs, M. Timkoc, A. Sapid and C. Visya, Synth. Met., 2010, 160, 65-71; (e) N. Khanduyeva, V. Senkovskyy, T. Beryozkina, M. Horecha, M. Stamm, C. Uhrich, M. Riede, K. Leo and A. Kiriy, J. Am. Chem. Soc., 2009, 131, 153-161.

25 P. Xu, X. J. Han, C. Wang, B. Zhang, X. H. Wang and H. L. Wang, Macromol. Rapid Commun., 2008, 29, 1392-1397. 26 L. Shao, J. W. Jeon and J. L. Lutkenhaus, Chem. Mater., 2012, 24, 181-189.

27 G. Nie, L. Y. Qu, J. K. Xu and S. S. Zhang, Electrochim. Acta, 2008, 53, 8351-8358.

28 W. Sun and X. Y. Chen, J. Power Sources, 2009, 193, 924-929.

29 H. Xu, J. L. Li, Z. J. Peng, J. X. Zhuang and J. L. Zhang, Electrochim. Acta, 2013, 90, 393-399.

30 B. K. Kuila, B. Nandan, M. Böhme, A. Janke and M. Stamm, Chem. Commun., 2009, 5749-5751.

31 M. S. Ramasamy, S. S. Mahapatra, H. J. Yoo, Y. A. Kim and J. W. Cho, ACS Macro Lett., 2014, 2, 4788-4794.

32 (a) K. H. Wong, M. H. Stenzel, S. Duvall and F. Ladouceur, Chem. Mater., 2010, 22, 1878-1891; (b) L. Li, Y. Zhong, C. Ma, J. Li, C. Chen, A. Zhang, D. Tang, S. Xie and Z. Ma, Chem. Mater., 2009, 21, 4977-4983; (c) L. Li, J. Li, Y. Zhong, C. Chen, Y. Ben, J. Gong and Z. Ma, J. Mater. Chem., 2010, 20, 5446-5453. 